\title{
Case Based Reasoning Support for Adaptive Finite Element Analysis: Mesh Selection for an Integrated System
}

\author{
Abid Ali Khan ${ }^{1}$, Imran Ali Chaudhry ${ }^{2} \&$ Ali Sarosh ${ }^{2}$ \\ ${ }^{1}$ Institute of Space Technology, Islamabad, Pakistan \\ ${ }^{2}$ National University of Sciences and Technology, Pakistan \\ Correspondence: Abid Ali Khan, Institute of Space Technology, Islamabad, Pakistan. Tel: 92-51-573-1527. \\ E-mail: khan.abid@ist.edu.pk
}

Received: December 27, 2013 Accepted: February 7, 2014 Online Published: April 8, 2014

doi:10.5539/apr.v6n3p21

URL: http://dx.doi.org/10.5539/apr.v6n3p21

\begin{abstract}
An Adaptive Finite Element Analysis Integrated System supported through application of Case Based Reasoning (CBR) methodology is being proposed in this paper. The approach is fruitful for selection of an initial mesh from a library of solutions to initiate analysis process, as already tested optimal mesh will have lesser refinement iterations. The optimal mesh distribution, represented by object-oriented method, can be easily adapted to the topology of new problem in same domain. An integrated and universal structural analysis system models human reasoning by forming solutions through the retrieval and adaptation of successful strategies used in the past. Basic insight of two distinct subjects along with resolution of involved issues and integration strategy for development of an intelligent system is elaborated here. The research explains an algorithm for case retrieval and mesh generation procedures based on the principles of mapping method.
\end{abstract}

Keywords: integrated system, case based reasoning, adaptive finite element analysis, mesh generation, mapping method, applied artificial intelligence, error estimation

\section{Introduction}

Over last few decades, Finite Element Method (FEM) has proved to be a strong numerical analysis method. FEM has eventually become an integral part or standard tool for design, synthesis and modeling process in virtually all-engineering sciences, such as, structures, thermodynamics, biomechanics, acoustics, materials, fracture mechanics, civil engineering, fault diagnosis and fluids dynamics etc (Borazjani, 2013; Bouaanani \& Lu, 2009; Cheng, White, \& Grosh, 2008; Dado \& Shpli, 2003; Fiedler, Hosseini, Belova, Murch, \& Öchsner, 2009; He \& Kwan, 2001; Mackerle, 2004; Noack, Rolfes, \& Tessmer, 2003; Smoljanović, Živaljić, \& Nikolić, 2013; Tabiei \& Wu, 2000; Wu, 2006; Zhang \& Gay, 2007). However, during its application in different disciplines, preparations associated with discretizing intricate multidimensional geometries hinder its wide spread recognition as an analysis tool (Burkhart, Andrews, \& Dunning, 2013; G. Nikishkov, Y. Nikishkov, \& Makeev, 2013). Importance of an optimum initial mesh in preparation for modeling or numerical analysis problems has been emphasized by the researchers time and again (Knupp, 2003). In addition to computational burden and issues of accuracy requirements, construction of an optimal and least expensive mesh is also a limitation being faced by operators of advanced Artificial Intelligence (AI) paradigms in this domain.

Since 80's when expert systems paced in to solving diverse science and engineering problems, researchers continuously proposed the applications of intelligence based assistance to structural analysis which was a good index in the growth of this field. A number of systems such as SACON (Sriram, Maher, \& Fenves, 1985), developed to support in selection of a suitable analysis strategy; FEASA (Taig, 1986), an effort that assisted in specification and modeling; PLASTRAN, FACS and CARTER (Cagan \& Genberg, 1987; Gregory \& Shephard, 1987; Reynier, 1986) some other examples in same category started to appear. Later in early 90's, EXPERT ASSISTANT (Labrie, Thilloy, Tanguy, \& Moll, 1994) and FEMES (Gong \& Xu, 1995) were developed to assist the users in this area of specialization. Pursuant to address these constraints, researchers ventured into application of CBR in different engineering domains (Bello-Tomás, González-Calero, \& Díaz-Agudo, 2004; Díaz-Agudo \& González-Calero, 2000; Díaz-Agudo, González-Calero, Recio-García, \& Sánchez-Ruiz-Granados, 2007; Hurley, 1994; Kumar \& Raphael, 1997; Lieven, Escamilla-Ambrosio, Bunniss, Burrow, \& Clare, 2009; Varma \& Roddy, 1999). 
Although applications discussed above had success in analysis of different type of structures but there are concerns that need addressing before advent of a universal approach (Fang, Luo, \& Tang, 2005). Many of existing systems are in engineering domains and deal with diagnosis, decision support, design, or planning but do not precisely resolve structural analysis issues (Bargmann, 1999; Grant, Harris, \& Moseley, 1996; Liao, 2004; Vong, Wong, \& Ip, 2011). The application of CBR in selection of optimal mesh for Adaptive Finite Element Analysis presented in this paper is being researched as an effort to develop a standard tool for engineering professionals in the structural analysis domain (Khan \& Ming, 1998).

\section{Adaptive Finite Element Analysis}

Over the last few decades wide spread acceptance of finite element method has compelled the analysts to recognize it as a standard analysis tool in engineering domain (Hinton, Rao, \& Özakça, 1991). Adaptive finite element is a problem solving technique that works on the principle of discretization, i.e., it places mesh over problem geometry and then approximates the solution on mesh nodes. Finite element model's accuracy greatly relies on mesh quality and number of nodes in the mesh distribution. In order to achieve required accuracy design of optimum meshes with varying element sizes is essential. Therefore it is said that number of nodes and accuracy is directly proportional to each other (Lim, Sohn, Lee, \& Im, 2010).

As the problems in real life are complex therefore adequate precision in the area of high variation may be acquired through a high node density (Wördenweber, 1984). In case of many real life problems it may not be apparent to user that how the solution would look like and where a fine mesh is needed. Owing to time and other constraints, typically there is a limit to the maximum number of nodes thus use of homogeneous fine mesh over the whole field is unacceptable. Instead a non-homogeneous mesh with varying compactness in accordance with localized requirements is preferred which requires high level of skill, hence, a tougher task for a common user.

The objective of this analysis technique is to normalize the discretization error. This is achieved by raising the number of degree of freedom in sections where model of preceding iteration was inadequate. The determination is to accomplish the extreme rate of convergence (Escobar, Montenegro, Montero, Rodríguez, \& González-Yuste, 2005; Lackner \& Mang, 2002; Nicolas \& Fouquet, 2013). The complete adaptive analysis process is shown in Figure 1, where error distribution for mesh in current iteration is numerically computed. These error computations deliver information on precision, solution reliability and guidance for mesh refinement in an effective style.

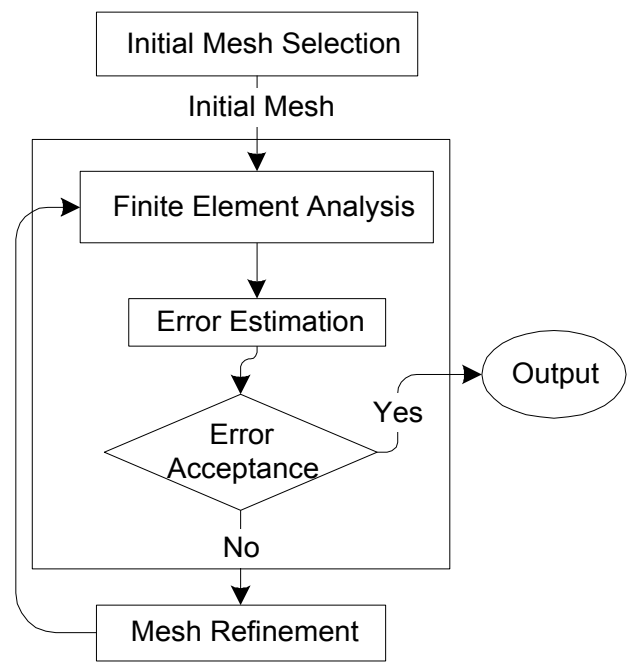

Figure 1. Adaptive finite element analysis process

\section{Mesh Generation Approaches}

It is important to understand the mesh generation schemes and process before any Artificial Intelligence paradigm is applied for assistance. Therefore, a brief review of the existing mesh generation schemes and their classification would be helpful to understand the case based mesh generation algorithm. A substantial research material has been published on the subject, which substantiates the significance and necessity of mesh generators. Reviews in 1973, 1980, 1988 and 1997 have been published on the existing approaches for grid generation. The 
first two did not compare and classify the schemes (Buell \& Bush, 1973; Thacker, 1980). Later, reviews published in 1988 and 1997 have covered the subject in a systematic way and classification provided in 1988 received more recognition for introducing the schemes (Hu, Zhang, Xiang, \& Zhou, 1997; Ho-Le, 1988).

A mesh generator produces a set of nodes and elements therefore existing strategies are being categorized in the order of creation of these sets. The strategies are divided into four main categories named as Mesh topology first, Nodes first, Adapted mesh template and other methods. The complete classification block diagram from review of 1988 is shown in Figure 2.

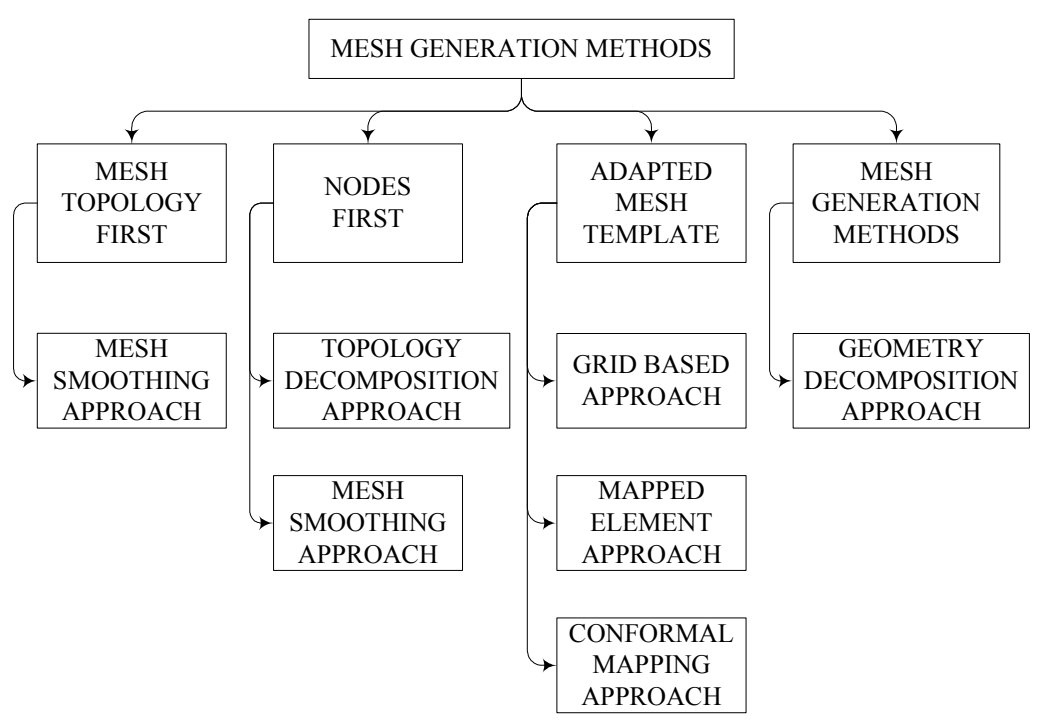

Figure 2. Classification scheme for mesh generation methods

Comparison of the grid generation schemes is a difficult task as access to the real systems is not available. Therefore, reliance on literature and inference of proficiencies for different schemes' is considered essential. The completely automatic methodologies are being compared and two partially automatic schemes, namely "Conformal mapping" and the "Mapped element" are not being considered. The four measures being used for comparison of mesh are element type, element shape, mesh density control and time efficiency. The detailed comparison of the four automatic methodologies is tabulated in Table 1.

Table 1. Comparison of the mesh generation approaches

\begin{tabular}{lllll}
\hline $\begin{array}{l}\text { COMPARISON } \\
\text { PARAMETERS }\end{array}$ & TOPOLOGICAL & NODE & GRID-BASED & $\begin{array}{l}\text { GEOMETRIC } \\
\text { DECOMPOSITION }\end{array}$ \\
\hline Quadrilateral & No & Yes & Yes & Yes \\
Brick & No & No & Relatively easy & No \\
Element shape & Poor & 2D good 3D fair & $\begin{array}{l}\text { Interior elements } \\
\text { excellent }\end{array}$ & 2D good 3D fair \\
Mesh density & No & Yes & Yes & Yes \\
Control & & & & \\
Time efficiency & $O\left(N^{2}\right)$ & $O(N)$ & $O(N)$ & Unknown \\
\hline
\end{tabular}

$(N=$ Number of nodes in the mesh). 


\section{Mesh Refinements}

In adaptive finite element analysis, refinement methods are categorized by researchers into two main classes that are; methods developed on experimental measures and finite element error approximations (Tezuka \& Okuda, 1988). In order to attain the optimum results a number of posteriori error estimation techniques have been (Cho \& Jun, 2004; Devloo, Tinsley Oden, \& Pattani, 1988; Gago, Kelly, Zienkiewicz, \& Babuska, 1983; Gatica, González, \& Meddahi, 2004; Houston, Schötzau, \& Wihler, 2006; Lee, Chiew, Lie, \& Nguyen, 2010; Maglo, Courbet, Alliez, \& Hudelot, 2012; Rank \& Roßmann, 1988; Schweitzer, 2009; Stewart \& Hughes, 1997; Warichet \& Legat, 1996; Yvonnet, Coffignal, Ryckelynck, Lorong, \& Chinesta, 2006). However, following four error estimation based adaptive re-meshing methods are being commonly used.

$h$-version.

The initial mesh elements are subdivided into smaller or combined into bigger elements for acquiring the required accuracy in a mesh.

p-version.

The polynomial order of interpolation function is changed while element geometry is kept constant.

$r$-version.

The nodes are relocated without increase in total number of nodes and elements.

hp-version.

$\mathrm{h}$ - and p- versions of refinement are simultaneously applied to get advantage from both strategies.

The application and choice from the above approaches depend on element type and needed engineering accuracy. Most commonly used method by scientists is h-version, whereas, $\mathrm{p}$ or hp-version are used for a very high accuracy requirements.

\section{Error Estimations}

Analysis of complex engineering problems with finite element method is normally faced by a conflict between solution accuracy and cost of computation. Over the years, to overcome this contradiction and acquire optimum precision for the solution, several adaptive approaches have evolved (Harutyunyan, Izsák, van der Vegt, \& Botchev, 2008; Hicken, 2012; Lee \& Zhou, 2004; Leicht \& Hartmann, 2010; Zhu \& Zienkiewicz, 1997). The two fundamental concerns involved in the methods of mesh refinement are "error estimation" and "mesh density control through suitable scheme". In order to quantify the extent of error in finite element analysis, error norms are used, energy norm $\|e\|$ being the most common. To evaluate this energy norm the two commonly used methodologies are as follows:-

\subsection{Residual Based Method}

Finite element solution provides an estimated and continuous displacement field over the domain; however, derivatives of this field are not continuous over element boundaries. This discontinuity implies strain which jumps across these boundaries. The error estimates based on this method (Babushka \& Reinhbolt, 1978; Kelly, Gago, Zienkiewicz, \& Babuska, 1983) utilize inter-element traction jump across element boundary and residual terms in the following governing equilibrium equation over element's interior to estimate an error:-

$$
L^{T} \widehat{\sigma}-b=0
$$

The energy norm $\|e\|$ is then given by

$$
\|e\|=\left(C_{1} \int_{\Omega} r^{T} r d \Omega+C_{2} \int_{I} J^{T} J d I\right)^{1 / 2}
$$

Where $\Omega$ is total domain, $\mathbf{C}_{\mathbf{1}}$ and $\mathbf{C}_{\mathbf{2}}$ constants, $\mathbf{I}$ total interface between elements, $\mathbf{J}$ inter-element traction and $\mathbf{r}$ are the residual forces that can be computed by using

$$
L^{T} \hat{\sigma}-b=r \neq 0
$$

Here the stresses $\sigma$ from Equation (1) have been approximated as $\sigma$ and obtained from standard relationship:

$$
\widehat{\sigma}=D B d
$$

In this relationship $\mathbf{D}$ is the elastic modulus matrix, $\mathbf{B}$ finite element strain-displacement matrix and $\mathbf{d}$ is the 
vector of nodal displacements.

\subsection{Best Guess Method}

The simple error estimator in its standard form uses projection techniques for defining a continuous stress filed over the domain. The use of a continuous stress field that eliminates the stress jumps over element boundary and specific "super convergent" points in the element where predicted results for stress are more accurate are the basic ideas in derivation of this error estimator. The error energy norm in this case is evaluated as,

$$
\|e\|=\left(\int_{\Omega}(\sigma-\widehat{\sigma})^{T} D^{-1}(\sigma-\widehat{\sigma}) d \Omega\right)^{1 / 2}
$$

In this scheme as exact solution is not accessible, therefore the stresses $\sigma$, are substituted by best guesses $\sigma^{*}$, such that $\sigma \approx \sigma^{*}$. The best guess in equation No 5 for standard iso-parametric elements can be determined by either least squares smoothing or loubignac iteration (Cantin, Loubignac, \& Touzot, 1978; Loubignac, Cantin, \& Touzot, 1977).

\subsubsection{Smoothing Procedures}

To find the component of the continuous stress field, it is approximated as:

$$
\sigma^{*}=\sum_{i} N_{\sigma_{i}} \bar{\sigma}_{i}
$$

Where $N_{\sigma_{i}}$ a continuous shape is function and $\bar{\sigma}_{i}$ is the smoothed nodal stress associated with node "I". The continuous stress $\sigma^{*}$ is determined by forcing the Galerkin-like condition

$$
\int_{\Omega} N_{\sigma_{i}}\left(\sigma^{*}-\hat{\sigma}\right) d \Omega=0
$$

To acquire a continuous stress field by Equations (6) and (7), it is assumed that the stress $\sigma^{*}$ is interpolated by the same shape function $\mathrm{Ni}$ as the individual displacement components. Thus,

$$
S \bar{\sigma}-q=0
$$

Where typical components may be written as

and

$$
\begin{aligned}
S_{i j} & =\int_{\Omega} N_{i}^{T} N_{j} d \Omega \\
q_{i} & =\int_{\Omega} N_{i} \hat{\sigma} d \Omega
\end{aligned}
$$

\subsubsection{Loubignac Algorithm}

While the traction jumps are eliminated between boundaries by use of continuous stress fields however, general equilibrium cannot be guaranteed as it does not satisfy the overall equilibrium conditions (Hinton, Özakça, \& Rao, 1991). The relationship for nodal equilibrium from governing equilibrium equations is derived as

$$
\int_{\Omega} B^{T} \sigma d \Omega-f=0
$$

\section{Application of Artificial Intelligence}

Recognizing the difficulties in FEM modeling, various developers are remedying the issues through application of AI and knowledge engineering techniques. As explained earlier, FEM is a potent and useful tool, but its effectiveness is hindered by the necessity to generate an appropriate initial mesh. To overcome this issue, which plays a vital role in acquisition of accurate solution through limited number of iterations, application of an advanced AI paradigm CBR is being proposed. CBR promises a technique to support modeling by reusing previous experiences in supporting the new situations (Ficet-Cauchard, Porquet, \& Revenu, 1999; Praehofer \& Kerschbaummayr, 1999; Surma \& Braunschweig, 1996).

Finite element model requires expert's experience in the related analysis domain as setting an appropriate grid on 
problem geometry is the difficult task faced by novice user. Most finite element analysis problems are inclined towards non-uniformity as solution varies in different parts of the domain. To attain appropriate accuracy, grid of higher node density may be indispensable in the areas of higher variation. Normally an upper limit to total number of nodes is customary due to time and other limitations, thus precluding the employment of a homogeneously fine grid over the whole domain. Therefore, non-uniformity of grid with varying density as per local needs become essential. Identifying a suitable density of mesh so that solution features are appropriately captured is therefore becomes most demanding task. Specifically in the adaptive finite element analysis correct generation of initial mesh greatly influences the rate of convergence and reduces the number of iterations required for an optimal solution. Here the experience of analyst and heuristics are prerequisite to choose best initial mesh.

As an effort to provide a truly robust adaptive finite element analysis system, this paper proposes application of CBR to assist in initial mesh selection. The devised scheme is not only simple and effective for Case Base Reasoner but also advantageous for fast origination of initial mesh.

\section{Case Based Reasoning}

This AI paradigm has given a well justified degree of optimism to AI's knowledge based decision support systems. The roots of Case-Based Reasoning traces back to last century (Schank, 1983), there are some other documented historical efforts that laid the foundations of CBR (Gentner, 1983; Smith \& Medin, 1981; Tulving, 1972; Wittgenstein, 1953). CYPRUS system might be called as a first Case-Based Reasoner (Kolodner, 1983a, 1983b). Since then hundreds of successful applications of CBR assisting different aspects in engineering and non-engineering domains have been reported (Auriol, Crowder, McKendrick, Rowe, \& Knudsen, 1999; Du, Liang, \& Sun, 2012; Li \& Sun, 2011; Li, Sun, J. Wu, \& X. -J. Wu, 2012; Lin \& Chen, 2011; Pereira \& Madureira, 2013; Ross, Fang, \& Hipel, 2002; Stéphane, Hector, \& Marc, 2010; Stéphane \& Marc, 2008).

CBR is a methodology of problem solving that uses a database, or case base (collection of earlier solutions) for solving new problems. The concerns for implementation of this approach are recalling, reusing and adapting of the relevant cases and saving the information of solved problem to case base (Voss, 1996). In general the procedure imitates the manner in which humans may resolve problems by re-using their earlier experience acquired from a similar situation where it has relevance to the new instance. A block diagram describing the CBR process is shown in Figure 3.

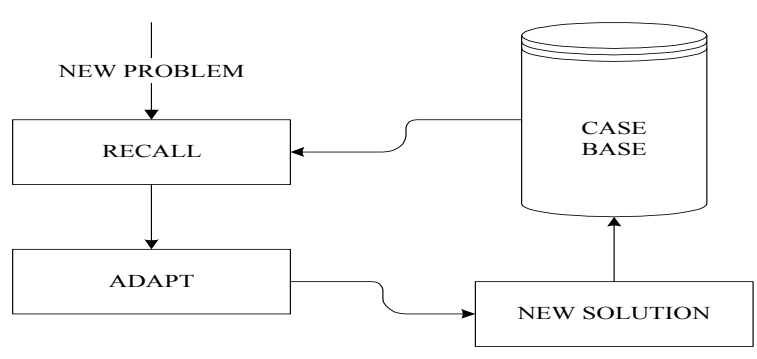

Figure 3. Case based reasoning model

\section{Integrated and Intelligent System Configuration}

CBR promises a technique to assist modeling process in finite element by reminding the analysts of prior experiences that can benefit new circumstances. A portion of the case base is a collection of cases that are real problems earlier solved by different experts, whereas, information for remaining portion comes from the knowledge, system gains by solving fresh problems as shown in Figure 4. The proposed system processes typical problem solutions into cases with machine learning methodology. Therefore, composition of case base is relatively easier than the rule base in customary expert systems. Case base becomes richer with use of proposed system through learning, thus enhancing the problem solving capacity.

User is only required to input the new problem's information such as, structure's geometrical attributes, load type and position, boundary condition, material and physical data as essential requirements of analysis. The system based on the attributes of new problem matches and retrieves the nearest resembling case from its case memory and generates the earlier analyzed mesh to initialize the adaptive analysis cycle. The solution acquired after 
reaching the required level of accuracy is stored in case base for future usage.

As an initial mesh plays a significant role in adaptive analysis process by complementing the convergence rate of finite element approximation. Thus analysts always emphasize on generating a high quality initial mesh. This demand gives rise to a need for improved initial mesh selection procedures that may serve as an equivalent to the competency of experts in the problem domain. Thus an integration of adaptive finite element analysis and case based reasoning technique is being realized to develop an "Intelligent and Integrated System" as seen in Figure 5.

A flow chart of CBR assisting adaptive finite element analysis is shown in Figure 6. Upon receipt of new problem definition the information is directed to the CBR inference engine which indexes its attributes and checks the case memory for availability of a similar prior incident. In the initial resemblance check, the cases with similar indices among the existing cases in case memory are selected for comprehensive matching. While in the detailed matching process, case with maximum similarity is retrieved for correction and adaptation. A nearest neighbor approach is utilized for selection of highest matching case. Once the case is corrected in accordance with input parameters, an initial grid pattern is generated on which the finite element computations are performed. The system thus produces a file consisting of an initial mesh pattern in the format suitable for use in customized or commercially available finite element analysis software. In case of a null return by the system, mesh generation module generates a default coarse initial mesh.

After setting an initial mesh system enters the analysis process and performs computations. The results so acquired are analyzed and errors are compared against preset accuracy. Upon satisfying the accuracy requirements the results are directed to output block, which are displayed and stored in the case memory simultaneously. In case, required accuracy is not achieved, mesh is refined following basic principle of adaptive finite element analysis based on errors estimates for the iteration. The process repeats, till the results converge and accuracy demand is satisfied.

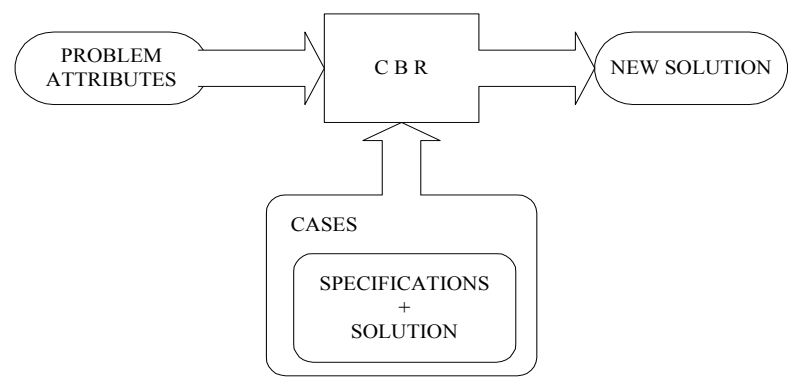

Figure 4. Case memory contents

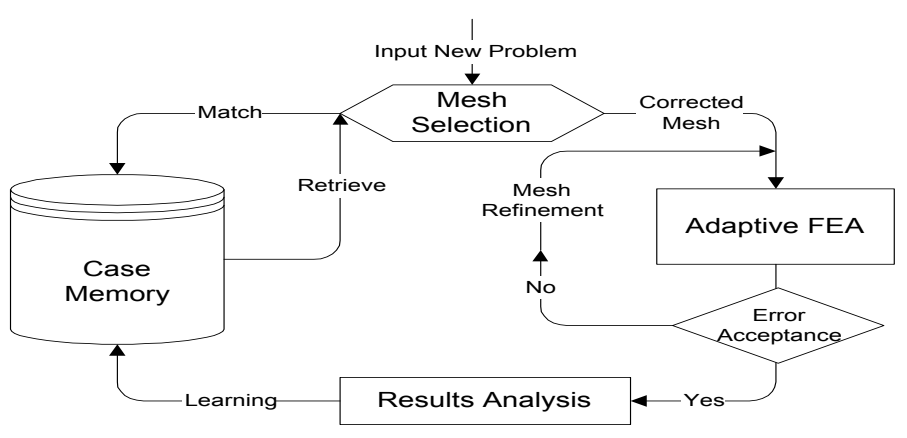

Figure 5. Integrated system framework 


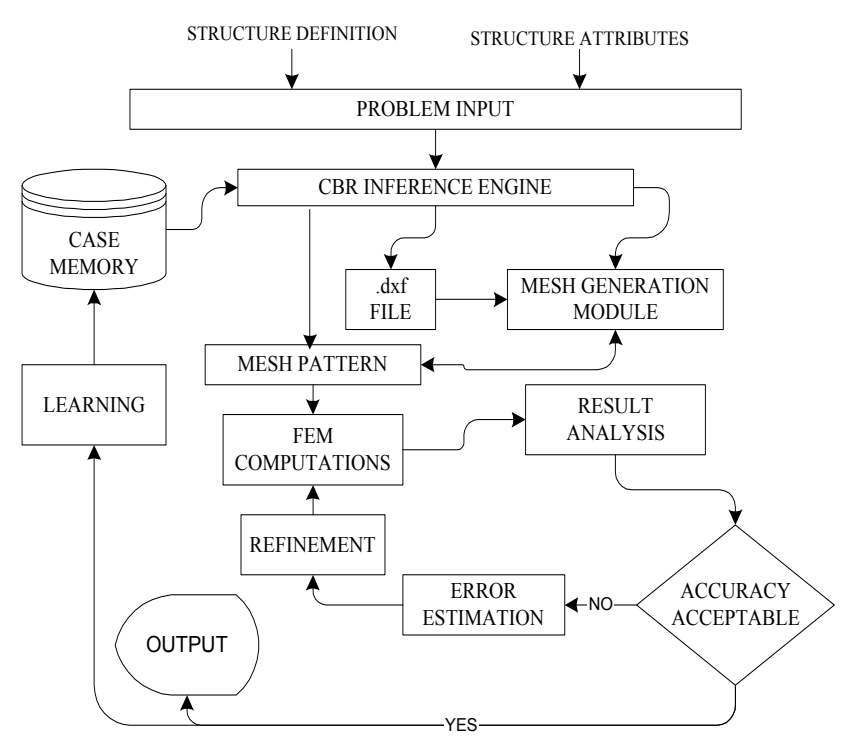

Figure 6. CBR system flow chart for adaptive FE analysis

The steps involved in the complete system designing after the recognition of problem and assessment of the full scope of the system can be explained as follows:

\subsection{Division of Task}

The multi-disciplinary task of system development is sub divided into hierarchical sub-problems. Sub-issues to resolve the issues of programming language selection, data flow path, knowledge acquisition strategy and implementation are deliberated in detail.

\subsection{Elements of CBR}

Case Based Reasoning concerns for system (Khan, 2001; Khan \& Azam, 2003), which is able to learn from past experiences, recognize similarities and retrieve them for new problem solving are:

\subsubsection{Case Representation}

A number of knowledge representation techniques such as, graph based, frame, multimedia representation and object oriented representations etc. exist and have been used by researchers in different problem domains. However, object oriented representation has been used for case representation in proposed system development.

\subsubsection{Case Indexing}

Attribute-Value pair scheme distinguished features of problem.

\subsubsection{Case Retrieval}

Nearest neighbor approach has been used for the matching and retrieval process of the stored cases.

\subsubsection{Case Adaptation}

Mapping technique is used to generate the initial mesh for new problem.

\subsubsection{Learning}

Successfully solved cases are stored in the case memory.

\subsection{Elements of Adaptive FEA}

Adaptive Finite Element Analysis requires that response quantities should be computed to the level of specified accuracy, therefore the concerns are:

\subsubsection{Error Estimator}

In order to compute reliable posteriori error estimates at reasonable cost, the algorithm for well-tested posteriori error estimator has been selected and realized for computing the errors (Zienkiewicz \& Zhu, 1987). 


\subsubsection{Refinement Strategy}

A simple " $h$-version" strategy has been used for refinement of mesh in successive iterations of adaptive finite element analysis process.

\subsection{Verification}

After resolving the sub-issues of "CBR" and "Adaptive FEM", "Reasoner" and "Analyzer" as shown in figure were separately developed and verified as shown in Figure 7.

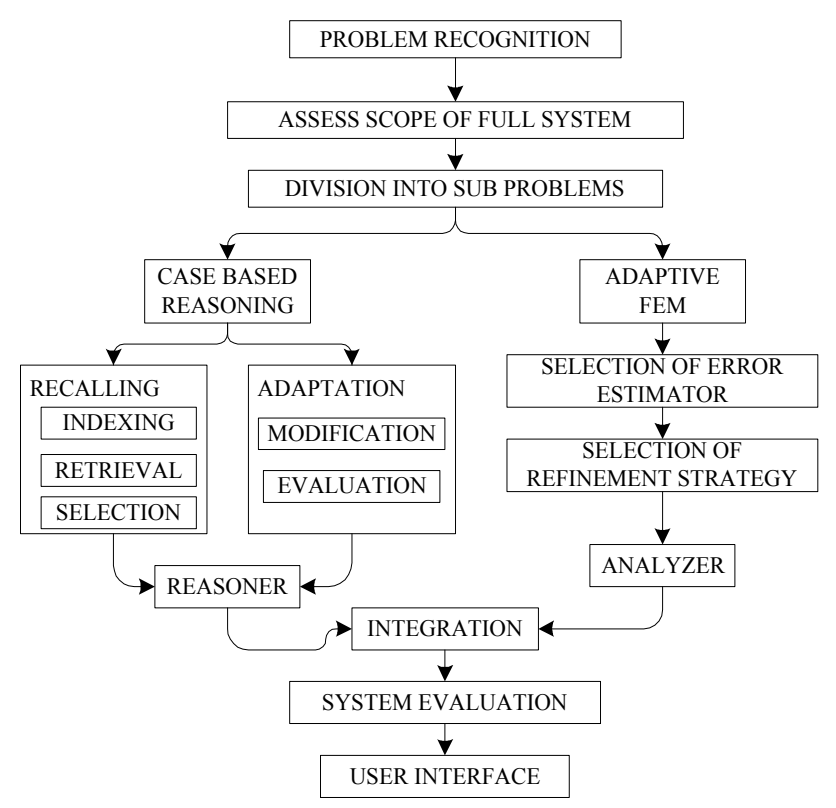

Figure 7. System realization

\subsection{Integration}

The "Reasoner" and "Analyzer" are integrated as a complete system.

\subsection{Performance Test}

System is tested for its performance by solving the typical two-dimensional structure analysis problems and it is evaluated for further improvements with continuation of research.

\subsection{User Interface}

Development of a user-friendly interface.

\section{Modules of Integrated System}

Five major functional modules of the system are developed after following the above elaborated seven steps process. The modules are shown in the follow chart of Figure 8 and briefly described in subsequent paragraphs. 


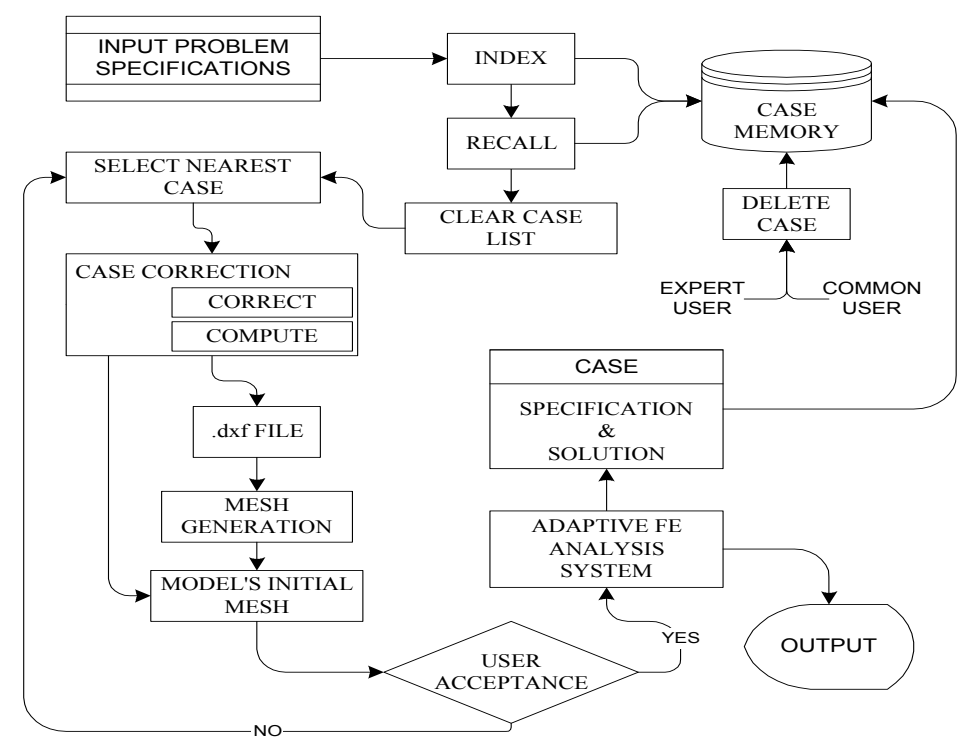

Figure 8. System flowchart

\subsection{Problem Description Module}

The module translates problem attributes from user defined problem to a message format, stored as a structure attributes file named "case.str" in accordance with case representation scheme. Therefore, this file encompasses information on number of boundaries in the generated geometric pattern, number of nodes, structure topology, load type and position, boundary condition and material data. A typical case named "C1" is shown in Figure 9, where "C" stands for a case with structure topology of three geometrical shapes as shown in Figure 10 and "1" signifies that it is first case in this category. It can be seen in Figure 9 that a total of six files named after the case name comprise complete information about the case as attribute-value pairs (Khan, X. Y. Ming, \& W. X. Ming, 1997).

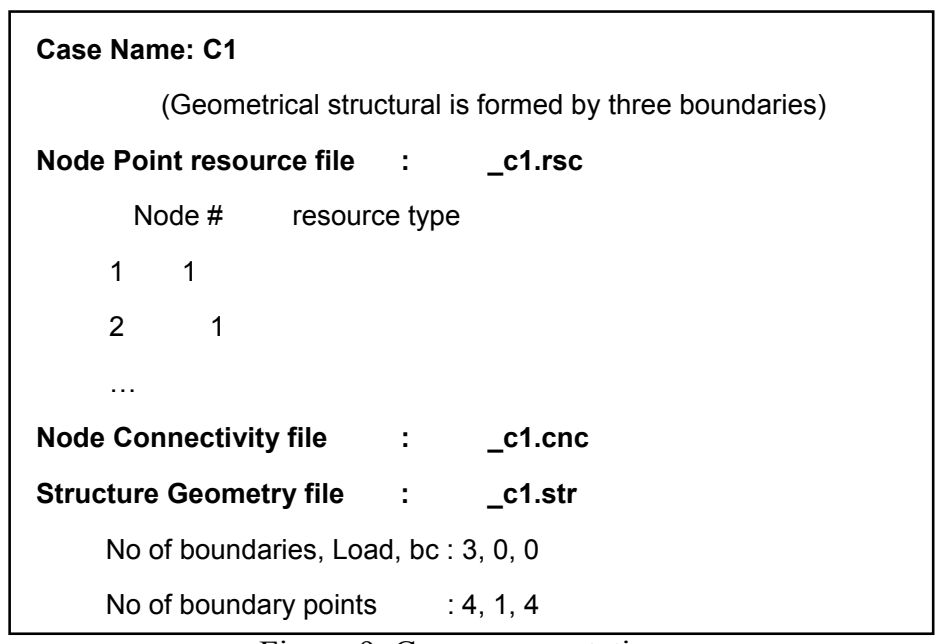

Figure 9. Case representation

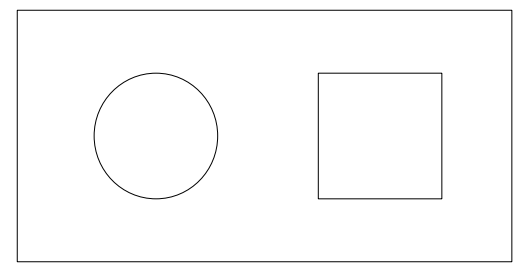

Figure 10. Structure topology 


\subsection{Case Recall or Retrieval Module}

This module indexes new case according to devised scheme. The module recalls the case base using associative recall method thus generating a list of nearest similar cases. These listed cases go through a similarity scrutiny process using nearest neighbor approach and weigh these cases as per degree of similarity. The highest weighed case is retrieved first and passes it for necessary correction to the adaptation module.

\subsection{Case Adaptation or Correction Module}

The case passed to this module after detailed matching undergoes necessary adaptation. The regions of the mesh that does not satisfy structure attributes of the new problem are re-meshed to generate initial mesh for the current problem. The module thus performs adaptation and correction tasks such as, removal of the node from the holes etc. and moving of nodes from outside of the boundary to within the structure topology.

\subsection{Mesh Generation Module}

Adaptive finite element analysis starts the finite element computations after receipt of the corrected initial mesh. This module produces a *.dxf file an accepted CAD format comprising information on initial mesh.

\subsection{Case Memory Management Module}

This module organizes the contents of case memory. It performs the functions of case storing, case deleting etc. In addition to the storage of cases through normal learning mechanism after successful solution of a problem, it also allows an expert user to store or delete a solution directly in the memory.

Basic principles of the involved theories and methodologies used for development of intelligent and integrated system have been discussed in the former part of this paper. Now, in the subsequent portion, it is important to go through the details of initial mesh generation mechanism which constitutes the most important part of this paper. Principle of mapping has been used for adaptation of an existing grid on the similar problem and features.

\section{Mapping Principle}

In plane " $Z$ ", a point expresses an independent variable " $z$ ", and the value of function " $w$ " expressed by a point in plane " $W$ ", then $w=f(z)$ can be regarded as mapping from point-group " $D$ " in plane " $Z$ " to the point group " $D$ " " in plane " $W$ ". If points are defined in the form of vectors $\vec{z}$ and $\vec{w}$ as, $x+y i$ and $u+v i$ respectively, then variable " $z$ " in accordance with corresponding relation acquires a unique value of the function.

$$
w=f(z)=u(x, y)+v(x, y) i
$$

\subsection{Transfinite Mapping Method}

The technique is a class of methods that establish curvilinear co-ordinate systems in an arbitrary domain. It generally defines an approximate entity (surface/volume), which will match a desired or true entity at a non-denumerable number of points. This property of the technique gives rise to the term "Transfinite Mapping". The property thus contrasts from iso-parametric mappings that match true surface at finite number of points, i.e. the interpolation points. In case of two dimensions, the transfinite mapping can exactly model all boundaries of the region, thus it does not introduce any geometric error. A concept of projector " $P$ " is introduced to describe the mappings. This projector is a linear operator that maps a true surface to an approximate one, established on inter-polatory constraints. Although there is a wide selection of possible projectors but discussion here is limited to a simple "Linear Lofting Projector". This projector implements a linear interpolation between two boundary curves, $\psi_{1}(u)$ and $\psi_{2}(u)$ :

$$
{ }_{P}^{[F]} \equiv P(u, v)=(1-v) \psi_{1}(u)+v \psi_{2}(u) ; 0 \leq u \leq 1,0 \leq v \leq 1
$$

Where $u$ is a normalized parametric co-ordinate along $\psi 1$ and $\psi 2$, and $v$ is a normalized co-ordinate that has a value of zero on $\psi 1$ and unity on $\psi 2$, as shown in Figure 11. It can be noted that the boundary of $P$ (Grant, Harris, \& Moseley, 1996; Varma \& Roddy, 1999; Vong, Wong, \& Ip, 2011) matches $F$ exactly along $\psi 1$ and $\psi 2$ that is a linear approximation. 


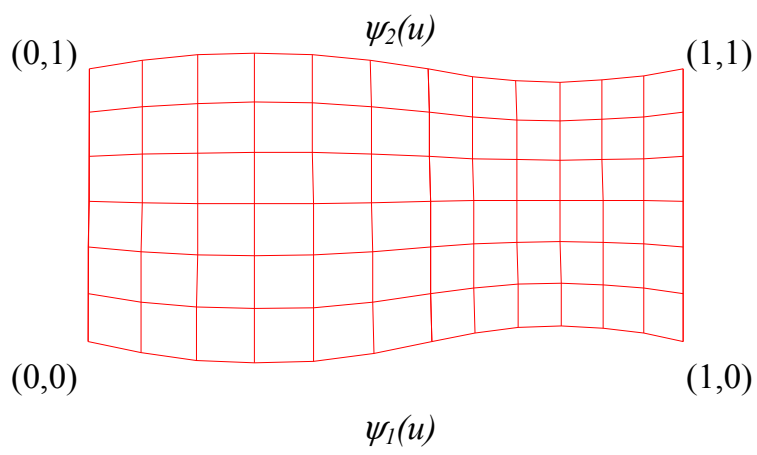

Figure 11. Lofting projector

\section{Matching, Retrieval and Adaptation}

All inclusive essential elements for a Case Based Reasoning system are not being elaborated in this paper and being published separately. Therefore, this paper mainly emphasizing mesh generation concerns. However, justice cannot be done without apprising the readers on remaining elements of CBR such as Matching, Retrieval and Adaptation; therefore same are being briefly touched here with an aim to cover these aspects in details in separate papers.

Matching of every case index compared to the target case is not only time consuming but also becomes expensive with the growth in case memory through learning. Case retrieval is therefore, employed with a filtering technique where cases are being segregated in the first step based on their names. In the second step segregated cases with same number of boundaries undergo detailed matching. A weighted feature scheme known as a nearest neighbor approach is used for detailed matching of segregated cases. Weights are assigned during the match process of structure geometry attribute file. All the parameters being numerical values are compared for a whole or partial match to determine the total weight Wtotal for a case. The procedure for comparison of target attribute $(a)$ and new case attribute $\left(a^{\prime}\right)$ is explained in subsequent paragraph.

If value $(a)=\operatorname{value}\left(a^{\prime}\right)$ then $W_{i}(a)=1$, this would mean a complete match and thus attribute $a$ is weighted as 1 or $100 \%$. Whereas, in case of value $(a) \neq \operatorname{value}\left(a^{\prime}\right)$ the attribute $a$ weighted as $W_{i}(a)=\operatorname{value}(a)-\operatorname{value}\left(a^{\prime}\right)$. This indicates that the weight $W_{i}$ for such as case will be less than 1 . The process is followed for all the attributes, after every comparison the assigned weight is added in the previous weight $W_{\text {previous }}$ in order to compute the $W_{\text {total }}$ as per Equation (13).

$$
W_{\text {total }}=W_{\text {previous }}+W_{i}\left(a^{\prime}\right)
$$

After computation of Wtotal during similarity analysis the cases are ranked in accordance with their assigned weights. Then from the ranked cases the case with highest weight is retrieved for reusing of its mesh on new problem's structure topology. Now for purpose of adaptation, mesh of selected case is checked for its validity by confirming that all the nodes for this mesh reside inside the outer boundary of new structure topology. Nodes found in an invalid region are automatically modified for moving them into the valid region before adaptation.

\section{Mesh Generation Algorithm}

As stated earlier that main purpose of this paper is to present the application of CBR on initial mesh selection for adaptive finite element analysis. Therefore, it is important to elaborate that mesh generation algorithm used here is different from any typical mesh generation techniques discussed in above paragraphs. The intelligent system uses Case Based approach, where an earlier successfully analyzed mesh pattern is matched, retrieved and adapted as an initial mesh for the structure under analysis to avoid generation a mesh from scratch. In order to adapt the selected mesh after matching and retrieval processes, mapping method is used to reuse selected mesh for analysis of the new problem. The following mesh generation algorithm is used for the system:

Procedure generate mesh()

$\{$ Read problem input file;

Convert data to case representation data files;

Identify indexing attribute; 
For each case(i) in case memory;

Match case name with indexing attribute;

Retrieve a set of cases that match indexing attribute;

Select nearest case (i);

Locate subfield origin points for problem as per case(i).sup;

// case (i).sup is a subfield division file of selected case( $i)$

Connect subfield origin points as per case(i).suc;

// case(i).sucis a connectivity file for subfields origin points of selected case(i)

Produce on-boundary \& on-circle points as per case(i).rsc;

// case(i).rscis a resource point file of selected case(i)

Repeat

Determine mapping function subfield( $k): M=f_{k}(Z), W=g_{k}(M)$;

Map meshed points from subfield(k) to medium plane $M$;

Map meshed points from medium plane M to subfield(j) of the problem;

Connect mesh points as per case(i).cnc;

// case(i).cncis a node connectivity file of selected case(i)

Until each subfield( $k$ ) in selected case(i) is mapped;

Repeat

Modify mesh to fit the problem structure;

Evaluate mesh;

Until solution is satisfactory;

\section{Example}

The geometric structure and its mesh pattern for a two dimensional rectangular plate with a hole in the center is shown in Figures 12(a) and (b). When the structure problems from the same class of two boundaries are input to the system for adaptive finite element analysis, mesh pattern is matched and retrieved from the case memory. This recalled grid is then mapped and adapted using above elaborated method to the different shaped plates. The example demonstrates the capability of the developed Case Based Reasoner in the problem domain. The selected mesh pattern is successfully mapped on a plate with an angular side Figure 12(c), tapered rectangular plate Figure 12(d), parallelogram shaped plate Figure 12(e), plate with two angular sides Figure 12(f) and a plate with all angular sides Figure 12(g). The example has successfully demonstrated the effectiveness of matching, retrieval and adaptation elements of the developed system.

\section{Conclusion}

Realizing the importance of initial mesh in an important field of engineering application of AI has been proposed to reduce the task of finite element user. The proposed system has shown an effective application of CBR in the adaptive finite element analysis domain through automatic mesh generation for initiation of the analysis process. The implementation and example-based evaluation of the designed system has shown an intelligent based approach for efficient analysis of engineering structures. The case base of the system continuously grows by accumulating information after every successful solution. The expert users of the system have also been permitted to directly input their experiences in the shape of earlier solved problem in case memory for future use. This powerful learning mechanism of CBR will always keep the case base updated. This will keep the system ready to deal with the real world problems, thus the changing world would not be able to make this knowledge obsolete.

Therefore, proposed application of CBR is helpful in greatly reducing the number of analysis cycles and computational burden thereby saving the extent of modeling work required by the user of adaptive finite element analysis. Furthermore, it also offers the theoretical basis and technical reserves for a continued future development in this domain. According to the success shown by the approach and development status, authors believe that further investigation and working on the same principles will evolve a more universal modeling methodology in near future. 


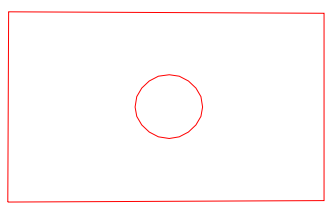

(a) Case geometry

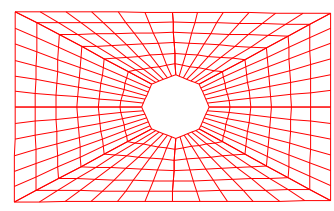

(b) Mesh pattern for case

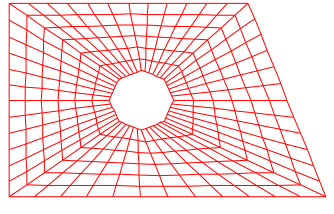

(c) Plate with angular side

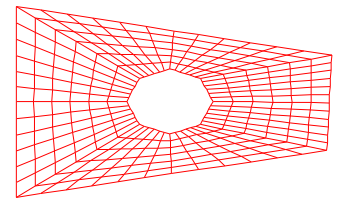

(d) Tapered rectangular plate

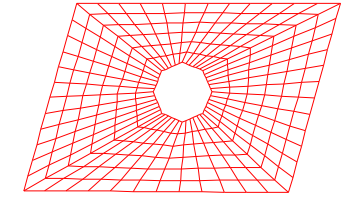

(e) Parallelogram shaped plate

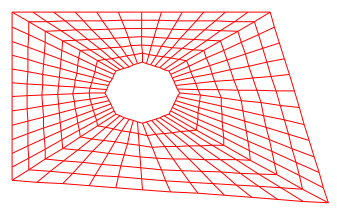

(f) Plate with two angular sides

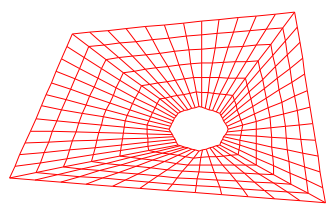

(g) Plate with all the angular sides

Figure 12. Adapted meshes from an existing case

\section{Future Directions}

Despite the satisfactory demonstration of the used approach, there are still issues that need further research. At present the system is successfully tested for solving two-dimensional analysis problems. This capability is being expanded to three-dimensional problem solving. Working on the same principles of mesh connectivity list model and node coordination information the system has a room for expansion into a full scope universal problem-solving tool. The possibility of effectively performing the tasks through incorporation of rules and frames with existing representation and development of a hybrid knowledge representation has not been completely ruled out. As we are aware that complex structure under different external environments, such as load condition and temperatures generally involve random and ambiguous information. Therefore, encapsulation of such information into case based reasoning system is another issue to be explored further. The uncertainty reasoning such as fuzzy logic is a good candidate for resolving such issues and will be considered during future work.

\section{References}

Auriol, E., Crowder, R. M., McKendrick, R., Rowe, R., \& Knudsen, T. (1999). Integrating case-based reasoning and hypermedia documentation: an application for the diagnosis of a welding robot at Odense steel shipyard. Engineering Applications of Artificial Intelligence, $12(6), \quad 691-703$. http://dx.doi.org/10.1016/S0952-1976(99)00040-8

Babushka, I., \& Reinhbolt, W. (1978). A-posteriori analysis for adaptive finite element computations. SIAM J. Numer. Anal., 15, 736-754.

Bargmann, R. (1999). Engineering Applications of Case-based Reasoning. Engineering Applications of Artificial Intelligence, $12(6)$.

Bello-Tomás, J. J., González-Calero, P. A., \& Díaz-Agudo, B. (2004). Jcolibri: An object-oriented framework for building cbr systems Advances in Case-Based Reasoning (pp. 32-46). Springer.

Borazjani, I. (2013). Fluid-structure interaction, immersed boundary-finite element method simulations of bio-prosthetic heart valves. Computer Methods in Applied Mechanics and Engineering, 257(0), $103-116$. http://dx.doi.org/10.1016/j.cma.2013.01.010

Bouaanani, N., \& Lu, F. Y. (2009). Assessment of potential-based fluid finite elements for seismic analysis of dam-reservoir systems. Computers \& Structures, 87(3), 206-224.

Buell, W., \& Bush, B. (1973). Mesh generation-A survey. Journal of Engineering for Industry, $95,332$. 
Burkhart, T. A., Andrews, D. M., \& Dunning, C. E. (2013). Finite element modeling mesh quality, energy balance and validation methods: A review with recommendations associated with the modeling of bone tissue. Journal of Biomechanics, 46(9), 1477-1488. http://dx.doi.org/10.1016/j.jbiomech.2013.03.022

Cagan, J., \& Genberg, V. (1987). PLASHTRAN: An expert consultant on two-dimensional finite element modeling techniques. Engineering with Computers, 2(4), 199-208.

Cantin, G., Loubignac, G., \& Touzot, G. (1978). An iterative algorithm to build continuous stress and displacement solutions. International Journal for Numerical Methods in Engineering, 12(10), 1493-1506.

Cheng, L., White, R. D., \& Grosh, K. (2008). Three-dimensional viscous finite element formulation for acoustic fluid-structure interaction. Computer Methods in Applied Mechanics and Engineering, 197(49-50), 4160-4172. http://dx.doi.org/10.1016/j.cma.2008.04.016

Cho, M., \& Jun, S. (2004). r-Adaptive mesh generation for shell finite element analysis. Journal of Computational Physics, 199(1), 291-316. http://dx.doi.org/10.1016/j.jpp.2004.02.007

Dado, M. H. F., \& Shpli, O. A. (2003). Crack parameter estimation in structures using finite element modeling. $\begin{array}{llll}\text { International Journal of Solids and Structures, } 40(20), & 5389-5406 .\end{array}$ http://dx.doi.org/10.1016/S0020-7683(03)00286-5

Devloo, P., Tinsley Oden, J., \& Pattani, P. (1988). An h-p adaptive finite element method for the numerical simulation of compressible flow. Computer Methods in Applied Mechanics and Engineering, 70(2), 203-235. http://dx.doi.org/10.1016/0045-7825(88)90158-2

Díaz-Agudo, B., \& González-Calero, P. A. (2000). An architecture for knowledge intensive CBR systems Advances in Case-Based Reasoning (pp. 37-48). Springer.

Díaz-Agudo, B., González-Calero, P. A., Recio-García, J. A., \& Sánchez-Ruiz-Granados, A. A. (2007). Building CBR systems with jCOLIBRI. Science of Computer Programming, 69(1), 68-75.

Du, Y., Liang, F., \& Sun, Y. (2012). Integrating spatial relations into case-based reasoning to solve geographic problems. Knowledge-Based Systems.

Escobar, J. M., Montenegro, R., Montero, G., Rodríguez, E., \& González-Yuste, J. M. (2005). Smoothing and local refinement techniques for improving tetrahedral mesh quality. Computers \& Structures, 83(28-30), 2423-2430. http://dx.doi.org/10.1016/j.compstruc.2005.03.022

Fang, X., Luo, H., \& Tang, J. (2005). Structural damage detection using neural network with learning rate improvement. Computers \& Structures, 83(25), 2150-2161.

Ficet-Cauchard, V., Porquet, C., \& Revenu, M. (1999). CBR for the management and reuse of image-processing expertise: a conversational system. Engineering Applications of Artificial Intelligence, 12(6), 733-747. http://dx.doi.org/10.1016/S0952-1976(99)00041-X

Fiedler, T., Hosseini, S. M. H., Belova, I. V., Murch, G. E., \& Öchsner, A. (2009). A refined finite element analysis on the thermal conductivity of perforated hollow sphere structures. Computational Materials Science, 47(2), 314-319. http://dx.doi.org/10.1016/j.commatsci.2009.08.007

Gago, D. S., Kelly, D., Zienkiewicz, O., \& Babuska, I. (1983). A posteriori error analysis and adaptive processes in the finite element method: Part II-Adaptive mesh refinement. International Journal for Numerical Methods in Engineering, 19(11), 1621-1656.

Gatica, G. N., González, M. a., \& Meddahi, S. (2004). A low-order mixed finite element method for a class of quasi-Newtonian Stokes flows. Part II: a posteriori error analysis. Computer Methods in Applied Mechanics and Engineering, 193(9-11), 893-911. http://dx.doi.org/10.1016/j.cma.2003.11.008

Gentner, D. (1983). Structure-mapping: A theoretical framework for analogy. Cognitive Science, 7(2), 155-170. http://dx.doi.org/10.1016/S0364-0213(83)80009-3

Gong, Y. N., \& Xu, Y. M. (1995). FEMES: Expert System for finite element modeling. Journal of Mechanical Strength, 2.

Grant, P. W., Harris, P. M., \& Moseley, L. G. (1996). Fault diagnosis for industrial printers using case-based reasoning. Engineering Applications of Artificial Intelligence, 9(2), 163-173. http://dx.doi.org/10.1016/0952-1976(96)00009-7

Gregory, B. L., \& Shephard, M. S. (1987). The generation of airframe finite element models using an expert system. Engineering with Computers, 2(2), 65-77. 
Harutyunyan, D., Izsák, F., van der Vegt, J. J. W., \& Botchev, M. A. (2008). Adaptive finite element techniques for the Maxwell equations using implicit a posteriori error estimates. Computer Methods in Applied Mechanics and Engineering, 197(17-18), 1620-1638. http://dx.doi.org/10.1016/j.cma.2007.12.006

He, X. G., \& Kwan, A. K. H. (2001). Modeling dowel action of reinforcement bars for finite element analysis of $\begin{array}{lllll}\text { concrete structures. } & \text { Computers } \& \quad \text { Structures, } & 79(6), & \text { 595-604. }\end{array}$ http://dx.doi.org/10.1016/S0045-7949(00)00158-9

Hicken, J. E. (2012). Output error estimation for summation-by-parts finite-difference schemes. Journal of Computational Physics, 231(9), 3828-3848. http://dx.doi.org/10.1016/j.jcp.2012.01.031

Hinton, E., Özakça, M., \& Rao, N. V. R. (1991). An integrated approach to structural shape optimization of linearly elastic structures. Part II: Shape definition and adaptivity. Computing Systems in Engineering, 2(1), 41-56. http://dx.doi.org/10.1016/0956-0521(91)90038-7

Hinton, E., Rao, N. V. R., \& Özakça, M. (1991). Mesh generation with adaptive finite element analysis. Advances in Engineering Software and Workstations, 13(5-6), 238-262. http://dx.doi.org/10.1016/0961-3552(91)90030-8

Ho-Le, K. (1988). Finite element mesh generation methods: A review and classification. Computer-Aided Design, 20(1), 27-38.

Houston, P., Schötzau, D., \& Wihler, T. P. (2006). An hp-adaptive mixed discontinuous Galerkin FEM for nearly incompressible linear elasticity. Computer Methods in Applied Mechanics and Engineering, 195(25-28), 3224-3246. http://dx.doi.org/10.1016/j.cma.2005.06.012

Hu, E. Q., Zhang, X. F., Xiang, W., \& Zhou, J. (1997). A review of mesh generation methods for finite element computation. Journal of Computer Aided Design \& Computer Graphics, 9(4), 378-383.

Hurley, N. (1994). A case based reasoning approach to mesh specification for adaptive finite element analysis. Mathematics and Computers in Simulation, 36(4), 381-388.

Kelly, D., Gago, D. S., Zienkiewicz, O., \& Babuska, I. (1983). A posteriori error analysis and adaptive processes in the finite element method: Part I-Error analysis. International journal for numerical methods in engineering, 19(11), 1593-1619.

Khan, A. A. (2001). Case Representation for Application of CBR in Structural Analysis Domain. Paper presented at the International Conference of Artificial Intelligence, Los Vegas, Nevada, USA.

Khan, A. A., \& Azam, F. (2003). CBR Assisting Computations in Structural Analysis. Paper presented at the Artificial Intelligence and Applications.

Khan, A. A., \& Ming, X. Y. (1998). Case-Based Retrieval in Adaptive Finite Element Analysis: Supporting Mesh Generation by Mapping Method. Paper presented at the 6th German Workshop on Case Based Reasoning, Berlin, Germany.

Khan, A. A., Ming, X. Y., \& Ming, W. X. (1997). Knowledge Representation in Case Based Reasoning for Finite Element Analysis. Paper presented at the 2nd Asian Pacific Conference on Aerospace Technology and Science (APCAT '97), Dun Huang Gansu, China.

Knupp, P. M. (2003). Algebraic mesh quality metrics for unstructured initial meshes. Finite Elements in Analysis and Design, 39(3), 217-241. http://dx.doi.org/10.1016/S0168-874X(02)00070-7

Kolodner, J. L. (1983a). Maintaining organization in a dynamic long-term memory. Cognitive Science, 7(4), 243-280. http://dx.doi.org/10.1016/S0364-0213(83)80001-9

Kolodner, J. L. (1983b). Reconstructive memory: A computer model. Cognitive Science, 7(4), 281-328. http://dx.doi.org/10.1016/S0364-0213(83)80002-0

Kumar, B., \& Raphael, B. (1997). CADREM: A case-based system for conceptual structural design. Engineering with Computers, 13(3), 153-164.

Labrie, R., Thilloy, C., Tanguy, P., \& Moll, G. (1994). An expert assistant to monitor finite element simulations. Mathematics and Computers in Simulation, 36(4), 413-422.

Lackner, R., \& Mang, H. A. (2002). Mesh generation and mesh refinement procedures for the analysis of

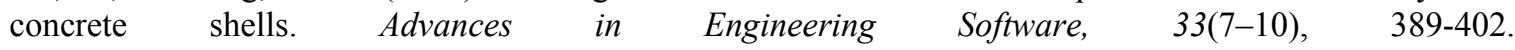
http://dx.doi.org/10.1016/S0965-9978(02)00054-6 
Lee, C. K., Chiew, S. P., Lie, S. T., \& Nguyen, T. B. N. (2010). Adaptive mesh generation procedures for thin-walled tubular structures. Finite Elements in Analysis and Design, 46(1-2), 114-131. http://dx.doi.org/10.1016/j.finel.2009.06.011

Lee, C. K., \& Zhou, C. E. (2004). On error estimation and adaptive refinement for element free Galerkin method: Part II: adaptive refinement. Computers \& Structures, 82(4-5), 429-443. http://dx.doi.org/10.1016/j.compstruc.2003.10.017

Leicht, T., \& Hartmann, R. (2010). Error estimation and anisotropic mesh refinement for 3d laminar aerodynamic flow simulations. Journal of Computational Physics, 229(19), 7344-7360. http://dx.doi.org/10.1016/j.jcp.2010.06.019

Li, H., \& Sun, J. (2011). On performance of case-based reasoning in Chinese business failure prediction from sensitivity, specificity, positive and negative values. Applied Soft Computing, 11(1), 460-467. http://dx.doi.org/10.1016/j.asoc.2009.12.005

Li, H., Sun, J., Wu, J., \& Wu, X.-J. (2012). Supply chain trust diagnosis (SCTD) using inductive case-based reasoning ensemble (ICBRE): The case of general competence trust diagnosis. Applied Soft Computing, 12(8), 2312-2321. http://dx.doi.org/10.1016/j.asoc.2012.03.029

Liao, T. W. (2004). An investigation of a hybrid CBR method for failure mechanisms identification. Engineering Applications of Artificial Intelligence, 17(1), 123-134. http://dx.doi.org/10.1016/j.engappai.2003.12.002

Lieven, N., Escamilla-Ambrosio, P., Bunniss, P., Burrow, S., \& Clare, L. (2009). Towards Wireless Sensor Usage and Health Monitoring of Helicopter Rotor Components.

Lim, J. H., Sohn, D., Lee, J. H., \& Im, S. (2010). Variable-node finite elements with smoothed integration techniques and their applications for multiscale mechanics problems. Computers \& Structures, 88(7-8), 413-425. http://dx.doi.org/10.1016/j.compstruc.2009.12.004

Lin, S.-W., \& Chen, S.-C. (2011). Parameter tuning, feature selection and weight assignment of features for case-based reasoning by artificial immune system. Applied Soft Computing, 11(8), 5042-5052. http://dx.doi.org/10.1016/j.asoc.2011.05.054

Loubignac, G., Cantin, G., \& Touzot, G. (1977). Continuous stress fields in finite element analysis. AIAA journal, $15(11), 1645-1647$.

Mackerle, J. (2004). Creep and creep fracture/damage finite element modelling of engineering materials and structures: An addendum. International Journal of Pressure Vessels and Piping, 81(5), 381-392. http://dx.doi.org/10.1016/j.ijpvp.2004.03.007

Maglo, A., Courbet, C., Alliez, P., \& Hudelot, C. (2012). Progressive compression of manifold polygon meshes. Computers \& Graphics, 36(5), 349-359. http://dx.doi.org/10.1016/j.cag.2012.03.023

Nicolas, G., \& Fouquet, T. (2013). Adaptive mesh refinement for conformal hexahedralmeshes. Finite Elements in Analysis and Design, 67(0), 1-12. http://dx.doi.org/10.1016/j.finel.2012.11.008

Nikishkov, G., Nikishkov, Y., \& Makeev, A. (2013). Finite element mesh generation for composites with ply waviness based on X-ray computed tomography. Advances in Engineering Software, 58(0), 35-44. http://dx.doi.org/10.1016/j.advengsoft.2013.01.002

Noack, J., Rolfes, R., \& Tessmer, J. (2003). New layerwise theories and finite elements for efficient thermal analysis of hybrid structures. Computers \& Structures, 81(26-27), 2525-2538. http://dx.doi.org/10.1016/S0045-7949(03)00300-6

Pereira, I., \& Madureira, A. (2013). Self-Optimization module for Scheduling using Case-based Reasoning. Applied Soft Computing, 13(3), 1419-1432. http://dx.doi.org/10.1016/j.asoc.2012.02.009

Praehofer, H., \& Kerschbaummayr, J. (1999). Case-based reasoning techniques to support reusability in a requirement engineering and system design tool. Engineering Applications of Artificial Intelligence, 12(6), 717-731. http://dx.doi.org/10.1016/S0952-1976(99)00043-3

Rank, E., \& Roßmann, A. (1988). Error estimate and adaptive mesh generation in finite element analyses. Computers and Geotechnics, 5(1), 74. http://dx.doi.org/10.1016/0266-352X(88)90035-3

Reynier, M. (1986). Interactions between Structural Analysis, Know-How and Chain of Reasoning used by the CARTER Expert System for Dimensioning. Reliability of Methods for Engineering Analysis, 533-544. 
Ross, S., Fang, L., \& Hipel, K. W. (2002). A case-based reasoning system for conflict resolution: Design and implementation. Engineering Applications of Artificial Intelligence, 15(3), 369-383.

Schank, R. C. (1983). Dynamic memory: A theory of reminding and learning in computers and people. Cambridge University Press.

Schweitzer, M. A. (2009). An adaptive hp-version of the multilevel particle-partition of unity method. Computer Methods in Applied Mechanics and Engineering, 198(13-14), 1260-1272. http://dx.doi.org/10.1016/j.cma.2008.01.009

Smith, E. E., \& Medin, D. L. (1981). Categories and concepts. Cambridge, MA: Harvard University Press.

Smoljanović, H., Živaljić, N., \& Nikolić, Ž. (2013). A combined finite-discrete element analysis of dry stone masonry structures. Engineering Structures, 52, 89-100. http://dx.doi.org/10.1016/j.engstruct.2013.02.010

Sriram, D., Maher, M. L., \& Fenves, S. J. (1985). Knowledge-based expert systems in structural design. Computers \& Structures, 20(1), 1-9.

Stéphane, N., Hector, R., \& Marc, L. L. J. (2010). Effective retrieval and new indexing method for case based reasoning: Application in chemical process design. Engineering Applications of Artificial Intelligence, 23(6), 880-894. http://dx.doi.org/10.1016/j.engappai.2010.03.005

Stéphane, N., \& Marc, L. L. J. (2008). Case-based reasoning for chemical engineering design. Chemical Engineering Research and Design, 86(6), 648-658.

Stewart, J. R., \& Hughes, T. J. R. (1997). An a posteriori error estimator and hp-adaptive strategy for finite element discretizations of the Helmholtz equation in exterior domains. Finite Elements in Analysis and Design, 25(1-2), 1-26. http://dx.doi.org/10.1016/S0168-874X(96)00059-5

Surma, J., \& Braunschweig, B. (1996). Case-base retrieval in process engineering: Supporting design by reusing flowsheets. Engineering Applications of Artificial Intelligence, 9(4), 385-391. http://dx.doi.org/10.1016/0952-1976(96)00036-X

Tabiei, A., \& Wu, J. (2000). Roadmap for crashworthiness finite element simulation of roadside safety structures. Finite Elements in Analysis and Design, 34(2), 145-157. http://dx.doi.org/10.1016/S0168-874X(99)00035-9

Taig, I. (1986). Expert aids to reliable use of finite element analysis. Reliability of Methods for Engineering Analysis, 457-474.

Tezuka, A., \& Okuda, O. (1988). An adaptive mesh refinement for the finite element method (Trial by rmethod). JSME International Journal, 31(1), 50-55.

Thacker, W. (1980). A brief review of techniques for generating irregular computational grids. International Journal for Numerical Methods in Engineering, 15(9), 1335-1341.

Tulving, E. (1972). Episodic and Semantic Memory 1. Organization of memory, 381-402.

Varma, A., \& Roddy, N. (1999). ICARUS: design and deployment of a case-based reasoning system for locomotive diagnostics. Engineering Applications of Artificial Intelligence, 12(6), 681-690. http://dx.doi.org/10.1016/S0952-1976(99)00039-1

Vong, C.-M., Wong, P.-K., \& Ip, W.-F. (2011). Case-based expert system using wavelet packet transform and kernel-based feature manipulation for engine ignition system diagnosis. Engineering Applications of Artificial Intelligence, 24(7), 1281-1294. http://dx.doi.org/10.1016/j.engappai.2011.07.002

Voss, A. (1996). How to solve complex problems with cases. Engineering Applications of Artificial Intelligence, 9(4), 377-384. http://dx.doi.org/10.1016/0952-1976(96)00033-4

Warichet, V., \& Legat, V. (1996). Adaptive hp-finite element viscoelastic flow calculations. Computer Methods in Applied Mechanics and Engineering, 136(1-2), 93-110. http://dx.doi.org/10.1016/0045-7825(96)01052-3

Wittgenstein, L. (1953). Philosophical investigations. Wiley-Blackwell.

Wördenweber, B. (1984). Finite element mesh generation. Computer-Aided Design, 16(5), 285-291. http://dx.doi.org/10.1016/0010-4485(84)90087-3

Wu, J.-J. (2006). Finite element analysis and vibration testing of a three-dimensional crane structure. Measurement, 39(8), 740-749. http://dx.doi.org/10.1016/j.measurement.2006.03.002 
Yvonnet, J., Coffignal, G., Ryckelynck, D., Lorong, P., \& Chinesta, F. (2006). A simple error indicator for meshfree methods based on natural neighbors. Computers \& Structures, 84(21), 1301-1312. http://dx.doi.org/10.1016/j.compstruc.2006.04.002

Zhang, L. T., \& Gay, M. (2007). Immersed finite element method for fluid-structure interactions. Journal of Fluids and Structures, 23(6), 839-857. http://dx.doi.org/10.1016/j.jfluidstructs.2007.01.001

Zhu, J. Z., \& Zienkiewicz, O. C. (1997). A posteriori error estimation and three-dimensional automatic mesh generation. Finite Elements in Analysis and Design, 25(1-2), 167-184. http://dx.doi.org/10.1016/S0168-874X(96)00037-6

Zienkiewicz, O. C., \& Zhu, J. Z. (1987). A simple error estimator and adaptive procedure for practical engineerng analysis. International Journal for Numerical Methods in Engineering, 24(2), 337-357.

\section{Copyrights}

Copyright for this article is retained by the author(s), with first publication rights granted to the journal.

This is an open-access article distributed under the terms and conditions of the Creative Commons Attribution license (http://creativecommons.org/licenses/by/3.0/). 